\title{
Random Forest Model For Predicting IDH1 Gene Expression Based on Volumetric Texture Parameters of WHO Grade II/III Glioma and Peritumoral EdemaRandom Forest Model for Predicting IDH1 Gene Expression Based on Volumetric Texture Parameters of WHO Grade II/III Glioma and Peritumoral Edema
}

\author{
Wenting Lan \\ Ningbo First Hospital \\ Zhan Feng \\ Zhejiang University School of Medicine First Affiliated Hospital \\ Yan Zhang \\ Ningbo Medical Center LiHuili Hospital \\ ZhengYa Zhao \\ Ningbo City First Hospital \\ Yi Huang \\ Ningbo City First Hospital \\ Xinzhong Ruan ( $\square$ Iwtrxz@163.com ) \\ Ningbo Hospital of Zhejiang University
}

Research article

Keywords: Glioma, Texture, Radiomics, Magnetic resonance imaging, IDH1

Posted Date: May 10th, 2021

DOI: https://doi.org/10.21203/rs.3.rs-496762/v1

License: (c) (i) This work is licensed under a Creative Commons Attribution 4.0 International License. Read Full License 


\section{Abstract}

Background: The incidence of Isocitrate dehydrogenase (IDH) gene mutation had closed contact with the development and prognosis of WHO grade II/III glioma. This study aims to establish and evaluate the predicting random Forest model for IDH1 gene mutation based on parenchyma and peritumoral edema ADC image texture parameters of WHO grade II/III glioma.

Materials and Methods: 146 patients (77 males and 69 females) with histologically confirmed anaplastic glioma were divided into training and validation groups in a ratio of 7:3 according to the requirements of Random Forest Model. The training group consisted of 102 patients (42 IDH1 mutant and 60 wild type) and the validation group included 44 patients (18 IDH1 mutant and 26 wild type). Conventional MRI features of two independent samples (IDH1 mutant and wild type) were evaluated by the Visually Accessible Rembrandt Images (VASARI) scoring system, Texture analysis (TA) of ADC image was based on the entire tumor volume and peritumoral edema and was used as Principal component analysis (PCA) to screen texture features labels. Random forest diagnosis models (VASARI+Tumor ${ }_{A D C} \square$ VASARI+Tumor $_{A D C}+$ Edema $_{A D C}$ ) were constructed on the basis of morphological single-factor variables, texture feature labels.

Result: The diagnostic accuracy of the random forest diagnosis model (VASARI+Tumor ADC) was 71.5\%, the specificity was $75.40 \%$, and the AUC was 0.769 , The model (VASARI+Tumor ${ }_{\text {ADC }}+$ peritumoral $^{2}$ edema ${ }_{\mathrm{ADC}}$ ) was $80.9 \%, 79.5 \%$, and 0.819 correspondingly.

Conclusion: The texture parameters of peritumoral edema ADC image were non-invasive markers to predict IDH1 mutational status and they have played a certain role in improving the efficiency of diagnostic model.

\section{Background}

Cerebral glioma has a high mortality and recurrence rate(1). It is believed

the pathogenesis is the cumulative result of environmental factors and complex genetic factors(2). Isocitrate dehydrogenase (IDH) is a key rate-limiting enzyme in the Tricarboxylic acid cycle(TCA) cycle which can provide energy and biosynthesis for the physical substance of cell metabolism (3). Parsons et al(4) studied polymorphism proteins in 22 glioblastoma (GBM) specimen cases, the results showed that about $12 \%$ were IDH1 codon 132 genes mutated case and the mutation was more common in young and neuroepithelial tumors in the secondary glioblastoma (WHOII-IIIlevel) patients. A large number of empirical studies showed that the incidence of IDH1 gene mutation was positively correlated with the overall survival rate of patients, and had close contact with the development and prognosis of glioma.(57). 2016 WHO classification of central nervous tumors have included IDH1 as molecular markers with a critical role in the selection of a treatment strategy and ha- important implications in patient outcome(8). More than $90 \%$ of glioblastomas belong to the IDH wild-type group, conversely, mutations in IDH 
represent a common (> 70\%) defining event in the development of low-grade glioma(9,10). More recently, the pan-mutant inhibitor BAY1436032 and MRK-A were tested in pre-clinical experiments using distinct dosing regimens to treat IDH1 R132H-expressing intracranial xenografts in BALB/c nude mice, the results show a small but statistically significant increase in animal survival in the treated group, and the survival rate increases with the concentration of the drug dose $(11,12)$. An increased emphasis on testing therapeutic strategies based on mutant IDH-dependent sensitivities may provide alternative therapeutic approaches that may benefit patients. Gliomas with IDH1 mutation present better responses to temozolomide and more sensitive to radiotherapy in-vitro than wild type counterparts which are especially appropriate for those unresectable glioma(13). Therefore, seeking specific diagnostic and prognostic biomarkers of IDH1 expression patterns shows particularly significant for II-III grade gliomas.

In addition to the proliferation and invasive growth of malignant tumor cells, peritumoral edema (PTE) is also an important factor affecting the therapeutic effect. PTE not only causes or increases the intracranial pressure and neurological impairment but also promotes the invasion and growth of tumor cells along the edema area. It increases the difficulty of total resection of tumors and has a high recurrence rate after surgery, which significantly increases the incidence of complications, disability , and mortality $(14,15)$. Diffusion-weighted magnetic resonance imaging (DWI) is a technique for measuring and imaging the diffusion of water molecules in vivo. The ADC (apparent diffusion coefficient) values have a good correlation with the composition of glioma cells and can be used for grading and evaluating the malignancy of glioma(16). Radiomics is an emerging technique that extracts unrevealed information by using a mathematical approach to assess gray-level intensity variations within a region of interest (ROI), a large high throughput quantitative features are automatically extracted(17)which helps to quantify the spatial distributions of intensities in images. It has shown well applied prospect in the field of oncology diagnosis, tumor heterogeneity quantifying, tumor tissue segmentation, tumor grading, classification, and predictions of treatment response and survival $(18,19)$. Although the expression of Ki67 has been positively correlated with the degree of $\operatorname{PTE}(20,21)$, whether peritumoral edema indicates the type of IDH1 expression in tumors remains to be studied. The study presented sought to investigate whether peritumoral edema radiomics parameters can predict IDH1 gene expression and to evaluate the diagnostic efficacy at the same time.

\section{Materials And Methods}

\section{Patients selection}

A retrospective study was performed at the XX Hospital of XX University. The patients from May 2015 to May 2018 were retrospectively analyzed with the following criteria: 1. Incipient brain glioma patients with WHOII-III grading (IDH1 immuno-histochemical results confirmed) 2. High-quality MRI imaging included T1WI, T2WI, DWI (ADC image), Flair and post-contrast T1WI series. 3.All the patients had not received the radiotherapy and chemotherapy treatment or given antitumor drug before surgery. Images could not be used for analysis were excluded. As a result, a total of 146 patients (77 males and 69 females) were enrolled in the study. Among them, 60 were IDH1 mutants and 86 were IDH1 wild type. The data were 
divided into training group and validation group according to the ratio of 7:3. The training group included 102 patients (42 IDH1 mutants and 60 wild type), and the validation group included 44 patients (18 IDH1 mutants and 26 wild type).

\section{MRI imaging acquisition}

All patients were examined following the same imaging acquisition protocol on a 1.5T MRI system (Philip Achieva Dutch) with a sixteen-channel head matrix coil. The conventional MR imaging protocols consisted of the following sequences: axial T1 weighted gradient-echo imaging (TR500ms;TE12ms), axial T2-weighted spin-echo imaging (TR5000ms;TE110ms), axial fluid-attenuated inversion recovery imaging (TR8400ms;TE120ms), and 3 orthogonal plane contrast-enhanced gradient-echo T1-weighted imaging scans (TR250ms;TE2.48ms) acquired with a dosage of $0.1 \mathrm{mmol} / \mathrm{kg}$. A high-pressure syringe was used for rapid injection from the elbow vein. The flow rate was $2 \mathrm{ml} / \mathrm{s}$. The section thickness $(5 \mathrm{~mm})$,

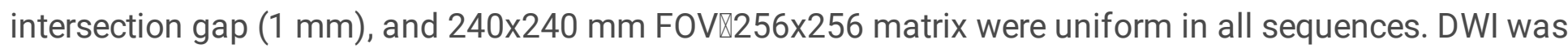
performed in the axial plane with a spin-echo echoplanar sequence before injection of contrast material. The imaging parameters used were as follows:TR4200ms;TE72.3ms, slice thickness:5mm, intersection gap: $1 \mathrm{~mm}$, FOV:220x220 mm. The b-values were 0 and $1000 \mathrm{~s} / \mathrm{mm}^{2}$ with diffusion gradient sencoded in the three orthogonal directions to generate 3 sets of diffusion-weighted images. Processing of the ADC image was generated automatically by the MR imaging system.

\section{Immunohistochemistry staining and Histopathological Dignosis}

Immunohistochemistry was performed on 5- $\mu$ m-thick sections from paraffin-embedded tumour specimens of all evaluated patients. IDH1R132H Mouse anti-Human Monoclonal Antibody (concentrated 1:50) was purchased from Fuzhou Maixin Biotechnology Co., Ltd. The samples for IDH1 mutation detection were repaired by EDTA antigen repair solution $(\mathrm{pH}=9.0)$ and citric acid buffer solution $(\mathrm{pH}=6.0)$. The samples were fixed by $4 \%$ neutral polyformaldehyde. EnVision two-step immunohistochemical staining was used for routine sampling, dehy-dration and paraffin-embedded tissue sections. The instrument used was LEICABOND-MAX automatic immunohistochemical staining instrument (LEICA, Germany). Normal autopsy brain tissue was used as negative control, and the positive section of known glioma IDH1 mutation was used as positive control. The staining results were determined by selecting 5 standard visual fields under 40-fold objective microscope. The expression of IDH1 mutant protein was localized in cytoplasm. Any number of brown cells appeared in the visual field of tumors, and the stained cells were regarded as positive mutations.

\section{Statistics analysis :}

Statistics analysis consists of three specific steps: 1. Screening statistical differences variables of general clinical data and conventional morphological features. 2. Texture lables $\bigotimes$ including peritumoral edema and tumor parenchyma冈acquisition and extraction. 3. Establishing and evaluating the preoperative diagnosis model of IDH1 gene type expression. Specific steps are as follows: 
1.Screening statistical differences variables of general clinical data and conventional morphological features:The general clinical data of the two groups were recorded, including gender and age.

Morphological scores based on VASARI were performed in all patients. All the data were analyzed by the R language (v.3.3.2 http://www.R-project.org) software. Shapiro-Wilk test was used to test the normality of quantitative data. The data conforming to normal distribution were expressed by ( $x \pm s)$. Those who did not conform to the normal distribution were expressed as median \pm quartile intervals. The quantitative data between IDH1 mutant and wild type groups were compared by independent sample $t$-test. Two samples were extracted according to the characteristic set of VASARI (Visual Accessible Rembrandt Images) of glioma. The Rembrandt Visual Accessible Images (VASARI) feature set is organized and planned by the radiologists of the Cancer Genome Atlas (TCGA) team (Location) based on MRI sequences T1WI, T2WI, FLAIR, DWI and enhanced T1WI conventional quantitative imaging features of gliomas which quantified the 30 qualitative descriptive features (included gender, age, location of tumors, maximum diameter冈peritumoral edema, intratumoral cystic degeneration, enhancement value of tumors $\rrbracket$ percentage of necrosis and extent of resection, etc.) and standardize the extraction process of quantitative features. In our experiment $₫$ we excluded the 26th, 27th, and 28th features which were not consistent with the purpose to predict IDH1 gene expression type before operation $\varangle$ but are more indicative of post-operative survival rate. Ultimately, 27 morphological features of tumors before the operation were evaluated. All the quantitative imaging features (Feature 1 to Feature 30, excluded the 26th, 27th and 28th ) were carried out by using single-factor analysis. Pearson's Chi-Square test was used for ranking data, and $t$-test for classifying variables was used for two independent samples. Independent predictors of IDH1 mutation were screened by multivariate Logistic regression $(\mathrm{P}<0.05)$.

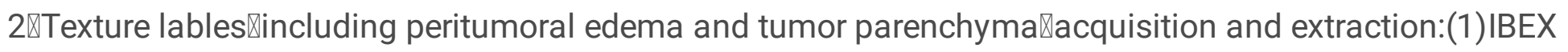
(v1.0 beta Dr. Laurence E, Court's Core Lab MD Anderson Cancer Center Houston TX77030) volume texture analysis software was performed based on MATLAB $2014 \mathrm{~b}$ platform. Volumetric ADC image texture parameters were drawn from the images of tumor parenchyma and peritumoral edema manually with ROI (Region of Interest) separately, and all volume texture parameters of both parenchyma and peritumoral edema were extracted, Figure1.The volume texture parameters of tumor parenchyma include six categories: Gradient Orient Histogram, Gray Level Cooccurrence Matrix, Intensity Direct, Intensity Histogram, Neighbor Intensity Difference and Shape with a total of 1733 texture features. The boundary of peritumoral edema refers to the FLAIR sequence. Because of subtracting the intermediate tumor entities, the second-order texture parameters cannot be evaluated. Therefore, we only choose histogrambased texture parameters to analyze. There were 50 texture parameters for edmea volumetric ADC image at last, concluding: Mean Absolute Deviation, Variance, Skewness, Kurtosis, percentiles (Percentile.01\%, Perc. $10 \%$, Perc. $50 \%$, Perc. $90 \%$, Perc. $99 \%$ ). The definition of texture parameters is explained as follows: 1.Degree of skewness: A class of statistics describing the symmetry of variable distribution, i.e. the degree of asymmetry of distribution relative to mean. Both positive and negative skewness show that the distribution is asymmetric. The larger the absolute value of skewness, the greater the skewness and the deviation from the normal distribution.(2)Kurtosis: statistics describing the shape steepness of a variable distribution, which reflects the degree of flatness or steepness compared with a normal distribution. The 
positive value is the steep peak, indicating that it is steeper than a normal distribution, while the negative value is the flat peak, indicating that it is flatter than a normal distribution. 3.Percentile: Describe the percentage of subjects observed below that percentile, reflecting small changes in the region of interest, closely related to the heterogeneity of lesions, the heterogeneity of lesions is large, the difference between high percentiles is greater, on the contrary, the difference between low percentiles is the smallest(22).

Texture analysis generates a large amount of information, and multivariate image analysis (MIA) could be a valuable tool for understanding and interpreting the data. The most essential part of the MIA procedure is principal component analysis (PCA).

Principal component analysis (PCA) was used to screen out texture labels from thousands of texture parameters which can indicate invisible pretreatment prognostic information. PCA is frequently used for reducing multi-dimensional data into a few principal segments and screening out latent variables that contain the majority of the variation in the data $(23,24)$.

All the extracted image texture parameters have seemed as variables in PCA statistics analysis. Variables with correlation coefficients greater than 0.999 were screened out by correlation analyses. The main components a cumulative contribution rate of more than $85 \%$ were selected, and the most influential feature vectors were found as the most important factors.

\section{Results}

1.The statistical results of the VASARI features set: There was no statistical difference between the two groups in age, gender ratio, location of tumors, maximum diameter. Statistical results showed that only peritumoral edema, cystic degeneration, and enhancement had a significant difference between the two groups $(P<0.001)$ (Table 1). Multivariate logistic regression showed that enhancement $(\mathrm{P}=0.015$, $\mathrm{OR}=2.775,95 \% \mathrm{Cl}: 1.231-5.750)$, cystic degeneration ( $\mathrm{P}=0.033, \mathrm{OR}=0.369,95 \% \mathrm{Cl}: 0.160-0.905)$ and peritumoral edema $(P=0.024,0 R=0.641,95 \% \mathrm{Cl}: 0) .0 .401-0.952)$ were independent predictors of IDHI mutant expression, which was consistent with the results of single-factor analysis(Table 2)

\section{Principal Component Analysis(PCA)Results:}

More than 1,000 correlations the texture parameters of ADC images of tumors parenchymal were extracted, mean clustering was used to classify and find 8 types of eigenvectors finally: Gradient OrientHistogram: (InterQuatile Range, Kurtosis), Graylevelcooccurrence Matrix (Auto-correlation, Cluster Prominence, Contrast) Sum Entropy Information Measurement Norm (Inverse Differential Norm) were characteristic texture parameter variables. The variables with a correlation coefficient greater than 0.999 were screened out, and principal component analyses were applied to these variables. When the principal component was two, these principal components had already explained $99.6 \%$ of the information (Table $3)$. 
Based on the VASARI feature set, three variables: peritumoral edema exist, cystic degeneration, and enhancement were combined with ADC texture label of tumor parenchyma were to be modeled. We established the random forest diagnostic Model $\triangle V A S A R I+$ Tumor $_{A D C}{ }^{\bigotimes}$. More than 500 trees are obtained to minimize the error. The validation data set was used to evaluate the effectiveness of the diagnosis model. The accuracy of the ROC curve was $82.7 \%$, the specificity was $68.40 \%$, and the AUC was $76.9 \%$ (Figure 2, 3).

The texture parameters of peritumoral edema histogram were obtained by using the correlation analysis method, and the variables with a correlation coefficient greater than 0.999 were screened out. A total of 32 variables were obtained and the principal components were analyzed. When the principal components were two, these principal components could explain $99.6 \%$ of the information. Among them, the parameters of $70 \%$ kurtosis and $80 \%$ skewness had characteristic significance (Table 4, Figures4, 5). Note: The cumulative contribution rate of PCA1 and PCA2 is $97.4 \%$ and $99.6 \%$ respectively.

When two texture labels of peritumoral edema $A D C$ image were added as new variables, the ROC curve of the model(VASARI+Tumor ${ }_{A D C}+$ Preitumoral edema $A D C$ ) was obtained (Figure 6), the results showed that the diagnostic accuracy of the model (VASARI+Tumor ${ }_{A D C}+$ Preitumoral edema $_{A D C}$ ) was $80.9 \%$, the specificity was $79.5 \%$, and the AUC increased to $81.9 \%$, which were slightly higher than that of the conventional MRI modality diagnosis model, and diagnostic accuracy was improved (Figure 5, 6). About $1 / 3$ of the out-of-bag data generated in the modeling data were verified repeatedly. The error rate of model classification and random forest out-of-bag data classification was stable at $10 \%$ in the end under different outcomes which indicated that the accuracy of the model was stable (Figure 7).

\section{Discussion}

The WHO classification of glioma has recently used molecular alterations in addition to histologic grading to define many tumor entities (26). Previous studies have focused on the imaging histology of the tumor parenchyma to identify the IDH status. IDH mutant glioma consistently demonstrated less aggressive imaging features than IDH wild-type glioma and showed frontal lobe predominance, less contrast enhancement, well-defined border, high apparent diffusion coefficient (ADC) value, and low relative cerebral blood volume ( $\mathrm{rCBV}$ ) value. However, few studies have focused on the relationship between peritumoral edema and tumor gene or molecular expression. This study attempts to use a new image analysis method to find the correlation between the two. Peritumoral edema of glioma is usually considered as angiogenic edema. The main pathogenesis is the increase of capillary permeability and the destruction of the blood-brain barrier. It was found that the rate of tumor vascular proliferation was highly related to tumor cell proliferation,capillary permeability is higher, prone to be more peritumoral edema(27). Quantitative apparent diffusion coefficient (ADC) values can reflect the tumor proliferative rate冈aggressiveness and the volume of the extracellular water compartment in cancer, it's a rapid and practical approach to assess glioma diffusivity and are sensitive to the changes of cell density, edema, and necrosis which usually be used to describe tumor dynamics(28). $\mathrm{Kim} \mathrm{H}$ et al(29)reported the average $A D C$ value of IDH1 wild type glioma were significantly higher than IDH1 mutant type. However, according 
to the reports of Yamashita et al(30), in the obtained IDH1 field there was no significant correlation between the mean $A D C$ values of the wild and the mutant type. Therefore, the traditional ADC value measuring analysis technology is controversial in reflecting exact gene information objectively as well as quantitatively.Lambin et al (17)proposed the use of computers to obtain high-throughput imaging features to correlate them with clinical information such as genotype, prognosis and therapeutic effect which could finally realize the prediction of relevant clinical information by constructing mathematical models. Texture analysis via Gray Level Co-occurrence Matrices (GLCM) is a method for extracting second-order statistical texture features in the images. Zhang et al (31)recently suggested that texture labels selected from SVM-RFE are more potential to identify IDH1 mutation, the model achieved accuracies of $86 \%$ (area under the curve $[A U C]=0.8830$ ) in the training cohort and $89 \%$ (AUC $=0.9231$ ) in the validation cohort. Su et al(32)examined 52 patients using multivariable logistic regression and found that the non-enhancing proportion of the tumor and the entropy of apparent diffusion coefficient (ADC) were found to possess higher prediction potential for IDH1 mutation with AUC of 0.918 and 0.724 , respectively. The tumor parenchyma $A D C$ texture analysis results in our study were consistent with these reports. According to the previous studies, peritumoral edema reflects the microvascular permeability, the activity of infiltration and proliferation of tumor冈the peritumoral edema is conducive to the movement of the cell matrix and adhesion molecules related to invasion, thus further promoting tumor cell infiltration $(33,34)$. Therefore, it is believed that the more severe the peritumoral edema of glioma is, the more obvious the tumor cell infiltration will be. Previous studies divided peritumoral edema into three regions: proximal, middle, and distal. In this study, the selected ROI concerns from overall assessment, the establishment of peritumoral edema all gray value texture parameters histogram, avoids the disadvantages of the limited local ROI. The results are in a consistency to the previous studies and are simple and convenient to interpret. Texture quantitative analysis was performed on routine MRI images of WHOII-III grade glioma and peritumoral edema of different types of IDH1, so as to improve the ability of differential diagnosis and provided a reference for the formulation of tumor resection and treatment plans. The results of this study indicate that Skewness $(70 \%)$ and kurtosis $(80 \%)$ of the histogram were the two important discrimination components that can identify the IDH1 gene expression type which was based on the PCA statistical analysis of the texture parameters in the peritumoral edema area. The degree of malignancy in glioma depends on the spread of tumor cells and the distribution of vascular endothelial growth factor囚VEGF囚major囚the more malignant the tumor, the more obvious the peripheral invasion, including the edema zone. Diffusion tensor imaging (DTI) had prompted that Peritumoral Brain Edema( PTBE) was an important role in differentiating benign and malignant Astro-cell tumors, a statistically significant difference had been established in ADC value and Fractional Anisotropy (FA) value in the PTBE region between benign and malignant tumor(34). Toh et al(35)compared the diffusivity (MD) value of PTBE regions between metastatic tumors and meningiomas and found that the mean MD value of metastatic turmors was significantly higher than meningiomas. Jissendi Tchofo $P$ (36)suggested that energy metabolism changes in the PTBE region is related to the extent of myelin sheath damage. For IDH1 wild type glioma, the worse the prognosis is, the greater the invasion of the tumor to the surrounding tissues, and the easier it is to relapse and metastasize. The tumor cell infiltration was also in the peritumoral edema area along with the diffuse infiltration of white matter 
fibers. As for the more obviously invasive growth of IDH1 glioblastoma, the component of peritumoral edema is more complicated and homogeneous. The more heterogeneity, the greater the kurtosis $\ a n d$ there were less infiltrating tumor cells. As the components were more homogeneous, the skewness was greater. The results of this study were found consistent with previous reports. Compared with the previous two-dimensional plane research, three-dimensional volumetric parameters are more comprehensive and objective.Construction of a comprehensive model that consists of both conventional VASARI parameters and ADC image texture parameters of glioma parenchyma and PTBE together had improved the preoperative prediction efficiency for the IDH1 gene type. The combination model yielded an AUC, sensitivity, and specificity of $0.819,88.5$, and $79.5 \%$ respectively. Here, there are still some deficiencies in our research $囚$ a single MRI sequence was used in texture analysis in our study and whether the multimodes MRI sequences are more comprehensive is still to be explored. As a corresponding study of IDH1 gene in different grade gliomas was not in part of the study. In addition, this was a retrospective study, with a possibility of population bias and the results may be tested by a prospective study. In conclusion the texture labels of the ADC image of peritumoral edema are non-invasive markers to predict IDH1 mutational status and they can improve the prediction efficiency of the preoperative IDH1 expression types model.

\section{Declarations}

\section{Informed consent}

Informed consent was obtained from all individual participants included in the study.

\section{Authors' contributions}

Study concept and design: RXZ; All the authors contributed to the data analysis and interpretation. LWT performed the statistical analysis; LWT, FZ and ZY were major contributors and contributed equally to writing the manuscript. ZY, ZZY provided the MRI data.HY provided the clinical data. All authors have read and approved the final manuscript.

\section{Availability of data and materials}

The datasets used and/or analyzed by the current study are available from the corresponding author on reasonable request.

\section{Ethics approval and consent to participate}

This retrospective study was approved by the Hospital Review Board.

Each patient was informed of the MRI risks and precautions and signed off before the examination. (Ningbo First Hospital Ethics Approval Commitee 2020-R175,2020-R176)

\section{Consent for publication}


Not applicable.

\section{Competing interests}

The authors declare that they have no conflicts of interest relevant to the contents of this manuscript.

\section{Acknowledgements}

None

\section{References}

1. Bondy ML, Scheurer ME, Malmer B, et al. Brain tumor epidemiology: consensus from the Brain Tumor Epidemiology Consortium. Cancer 2008;113:1953-1968.

2. Omuro A, DeAngelis LM. Glioblastoma and other malignant gliomas: a clinical review. JAMA 2013;310:1842-1850.

3. Dang L, White DW, Gross S, et al. Cancer-associated IDH1 mutations produce 2-hydroxyglutarate. Nature 2010;465:966.

4. Parsons DW, Jones $S$, Zhang $X$, et al. An integrated genomic analysis of human glioblastoma multiforme. Science 2008;321:1807-1812.

5. Watanabe T, Nobusawa S, Kleihues P, et al. IDH1 mutations are early events in the development of astrocytomas and oligodendrogliomas. Am J Pathol 2009;174:1149-1153.

6. Shin JH, Lee YS, Hong YK, et al. Correlation between the prognostic value and the expression of the stem cell marker CD133 and isocitrate dehydrogenase 1 in glioblastomas. J Neurooncol 2013;115:333-341.

7. Zhang Q, Liu K, Yang W, et al. Detection of IDH1 and IDH2 Mutation in Formalin-fixed Paraffinembedded Gliomas Using Allele-specific COLD-PCR and Probe Melting Curve Analysis. Appl Immunohistochem Mol Morphol 2018;26:e93-93e100.

8. Komori T. The 2016 WHO Classification of Tumours of the Central Nervous System: The Major Points of Revision. Neurol Med Chir (Tokyo) 2017;57:301-311.

9. Dang L, White DW, Gross S, et al. Cancer-associated IDH1 mutations produce 2-hydroxyglutarate. Nature 2009;462:739-744.

10. Rossetto M, Ciccarino P, Boisselier B, et al. Metabolism of glioma and IDH1/IDH2 mutations. Rev Neurol (Paris) 2011;167:699-703.

11. Pusch S, Sahm F, Meyer J, et al. Glioma IDH1 mutation patterns off the beaten track. Neuropathol Appl Neurobiol 2011;37:428-430.

12. Kopinja J, Sevilla RS, Levitan D, et al. A Brain Penetrant Mutant IDH1 Inhibitor Provides In Vivo Survival Benefit. Sci Rep 2017;7:13853. 
13. Cairncross JG, Wang M, Jenkins RB, et al. Benefit from procarbazine, lomustine, and vincristine in oligodendroglial tumors is associated with mutation of IDH. J Clin Oncol 2014;32:783-790.

14. Liang HT, Chen WY, Lai SF, et al. The extent of edema and tumor synchronous invasion into the subventricular zone and corpus callosum classify outcomes and radiotherapy strategies of glioblastomas. Radiother Oncol 2017;125:248-257.

15. Bussat A, Proisy $M$, Bruneau $B$, et al. Edema of the optic tract in patients with tumors of the sellar region: clinical and visual implications in the pediatric population. J Neurosurg Pediatr 2018;21:516522.

16. Rowley HA, Grant PE, Roberts TP. Diffusion MR imaging. Theory and applications. Neuroimaging Clin N Am 1999;9:343-361.

17. Lambin P, Rios-Velazquez E, Leijenaar R, et al. Radiomics: extracting more information from medical images using advanced feature analysis. Eur J Cancer 2012;48:441-446.

18. Georgiadis P, Cavouras D, Kalatzis I, et al. Enhancing the discrimination accuracy between metastases, gliomas and meningiomas on brain MRI by volumetric textural features and ensemble pattern recognition methods. Magn Reson Imaging 2009;27:120-130.

19. Assefa $D$, Keller $H$, Ménard $C$, et al. Robust texture features for response monitoring of glioblastoma multiforme on T1-weighted and T2-FLAIR MR images: a preliminary investigation in terms of identification and segmentation. Med Phys 2010;37:1722-1736.

20. Wick W, Küker W. Brain edema in neurooncology: radiological assessment and management. Onkologie 2004;27:261-266.

21. Carlson MR, Pope WB, Horvath $S$, et al. Relationship between survival and edema in malignant gliomas: role of vascular endothelial growth factor and neuronal pentraxin 2. Clin Cancer Res 2007;13:2592-2598.

22. DeWitt JC, Mock A, Louis DN. The 2016 WHO classification of central nervous system tumors: what neurologists need to know. Curr Opin Neurol 2017;30:643-649.

23. Brynolfsson P, Nilsson D, Henriksson R, et al. ADC texture-an imaging biomarker for high-grade glioma. Med Phys 2014;41:101903.

24. Hess AS, Hess JR. Principal component analysis. Transfusion 2018;58:1580-1582.

25. Erickson BJ, Korfiatis P, Akkus Z, et al. Machine Learning for Medical Imaging. Radiographics 2017;37:505-515.

26. Comprehensive genomic characterization defines human glioblastoma genes and core pathways. Nature 2008;455:1061-1068.

27. Pore N, Liu S, Haas-Kogan DA, et al. PTEN mutation and epidermal growth factor receptor activation regulate vascular endothelial growth factor (VEGF) mRNA expression in human glioblastoma cells by transactivating the proximal VEGF promoter. Cancer Res 2003;63:236-241.

28. Abdalla G, Sanverdi E, Machado PM, et al. Role of diffusional kurtosis imaging in grading of brain gliomas: a protocol for systematic review and meta-analysis. BMJ Open 2018;8:e025123. 
29. Kim H, Kim S, Lee HH, et al. In-Vivo Proton Magnetic Resonance Spectroscopy of 2-Hydroxyglutarate in Isocitrate Dehydrogenase-Mutated Gliomas: A Technical Review for Neuroradiologists. Korean J Radiol 2016;17:620-632.

30. Yamashita K, Hiwatashi A, Togao 0, et al. MR Imaging-Based Analysis of Glioblastoma Multiforme: Estimation of IDH1 Mutation Status. AJNR Am J Neuroradiol 2016;37:58-65.

31. Zhang B, Chang K, Ramkissoon S, et al. Multimodal MRI features predict isocitrate dehydrogenase genotype in high-grade gliomas. Neuro Oncol 2017;19:109-117.

32. Burger PC, Vogel FS, Green SB, et al. Glioblastoma multiforme and anaplastic astrocytoma. Pathologic criteria and prognostic implications. Cancer 1985;56:1106-1111.

33. Law M, Cha S, Knopp EA, et al. High-grade gliomas and solitary metastases: differentiation by using perfusion and proton spectroscopic MR imaging. Radiology 2002;222:715-721.

34. van Westen D, Lätt J, Englund E, et al. Tumor extension in high-grade gliomas assessed with diffusion magnetic resonance imaging: values and lesion-to-brain ratios of apparent diffusion coefficient and fractional anisotropy. Acta Radiol 2006;47:311-319.

35. Toh $\mathrm{CH}$, Wong AM, Wei KC, et al. Peritumoral edema of meningiomas and metastatic brain tumors: differences in diffusion characteristics evaluated with diffusion-tensor MR imaging. Neuroradiology 2007;49:489-494.

36. Jissendi Tchofo P, Balériaux D. Brain (1)H-MR spectroscopy in clinical neuroimaging at 3T. J Neuroradiol 2009;36:24-40.

\section{Tables}

Table 1: Single factor analysis of VASARI features of conventional MR

\begin{tabular}{|llll|}
\hline Characteristics & IDH1 mutant & IDH1wild-type & Pvalue \\
\hline Edema & & & $<0.001$ \\
\hline None & 12 & 4 & \\
\hline Range $\geq 2 \mathrm{~cm}$ & 5 & 29 & \\
\hline 2cm & 3 & 2 & $<0.001$ \\
\hline Cystic degeneration & & & \\
\hline Exist & 4 & 31 & $<0.001$ \\
\hline None & 16 & 4 & \\
\hline Enhancment & & & \\
\hline None & 15 & 0 & \\
\hline Ehanced(wreath like $\rrbracket$ & 5 & 35 & \\
\hline
\end{tabular}


Table 2 :Multivariate logistic regression analysis:

\begin{tabular}{|lllll|}
\hline Selected variables & regression coefficient & OR value & $95 \%$ confidence interval & $P$ value \\
\hline Edema & 0.965 & 0.640 & $0.401 \sim 0.952$ & 0.024 \\
\hline Cyst degeneration & -0.946 & 0.369 & $0.160 \sim 0.905$ & 0.033 \\
\hline Enhancment & -0.540 & 2.775 & $1.231 \sim 5.750$ & 0.015 \\
\hline
\end{tabular}

Table 3: Feature variables of tumor mass

\begin{tabular}{|llll|}
\hline Feature variables & & PCA1 & PCA2 \\
\hline GradientOrientHistogram & InterQuatileRange & 0.974 & 0.558 \\
\hline Graylevelcooccurence Matrix & Kurtosis & 0.022 & 0.853 \\
& Auto-Correlation & 0.025 & 0.284 \\
\hline & ClusterProminence & 0.003 & 0.098 \\
\hline & Contrast & 0.013 & 0.063 \\
\hline & SumEntropy & 0.475 & 0.390 \\
\hline & InformationMeasurecorr & 0.195 & 0.250 \\
\hline & InverseDiffMomentNorm & 0.132 & 0.0205 \\
\hline
\end{tabular}

Table 4: Feature variables of peritumoral edema

\begin{tabular}{|lll|}
\hline Feature variables & PCA1 & PCA2 \\
\hline Kurt $_{\text {ADC (p70) }}$ & 0.177 & -0.253 \\
\hline Skew $_{\mathrm{ADC}(\mathrm{p} 80)}$ & 0.176 & -0.229 \\
\hline
\end{tabular}

\section{Figures}
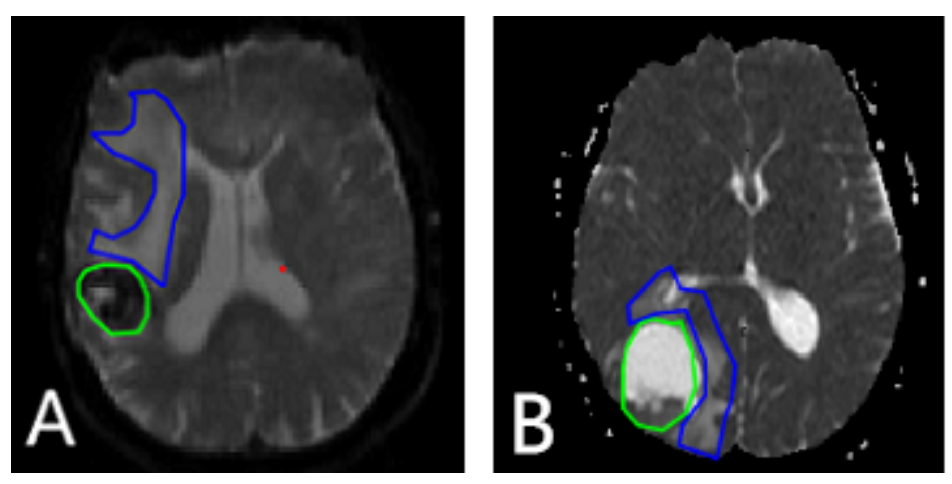
Figure 1

With different color labeling, the tumors and edema areas were delineated layer by layer on the crosssectional axial images. The software automatically synthesizes three-dimensional images, obtains the volume images of tumors and retained edema areas, and collects the overall data of tumors by the

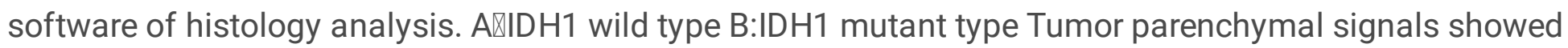
different manifestations on ADC diagram with different IDH1 type

\section{Image not available with this version}

Figure 2

Quantitative Error Graph for Selecting Random Trees of model\VASARI+TumorADC》 
OOB ROC Curve Random Forest (VASARI+TumorADC)

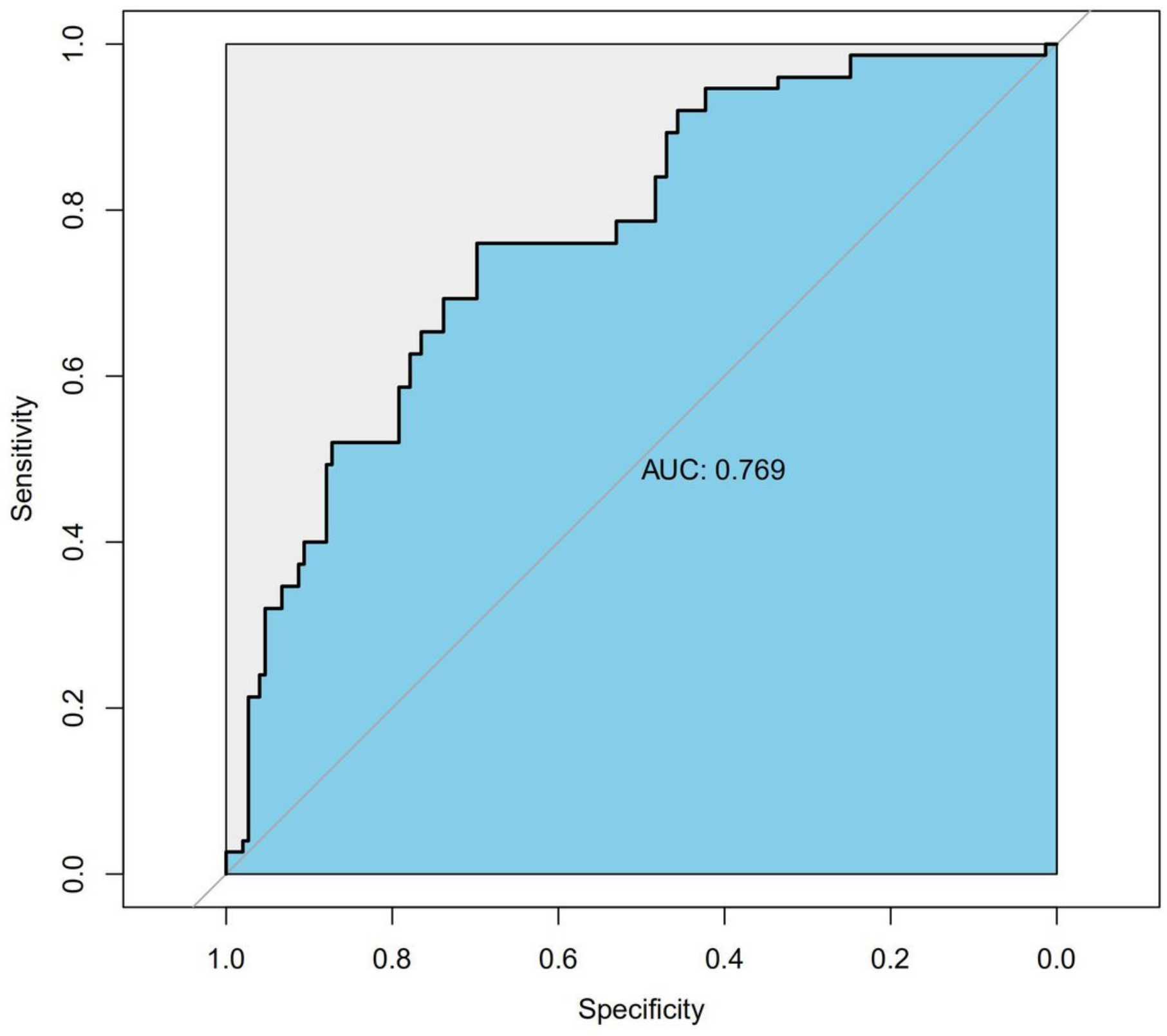

Figure 3

Random forest dignositic model $\varangle$ VASARI+TumorADC $\triangle$ The area under ROC curve is $76.9 \%$. 


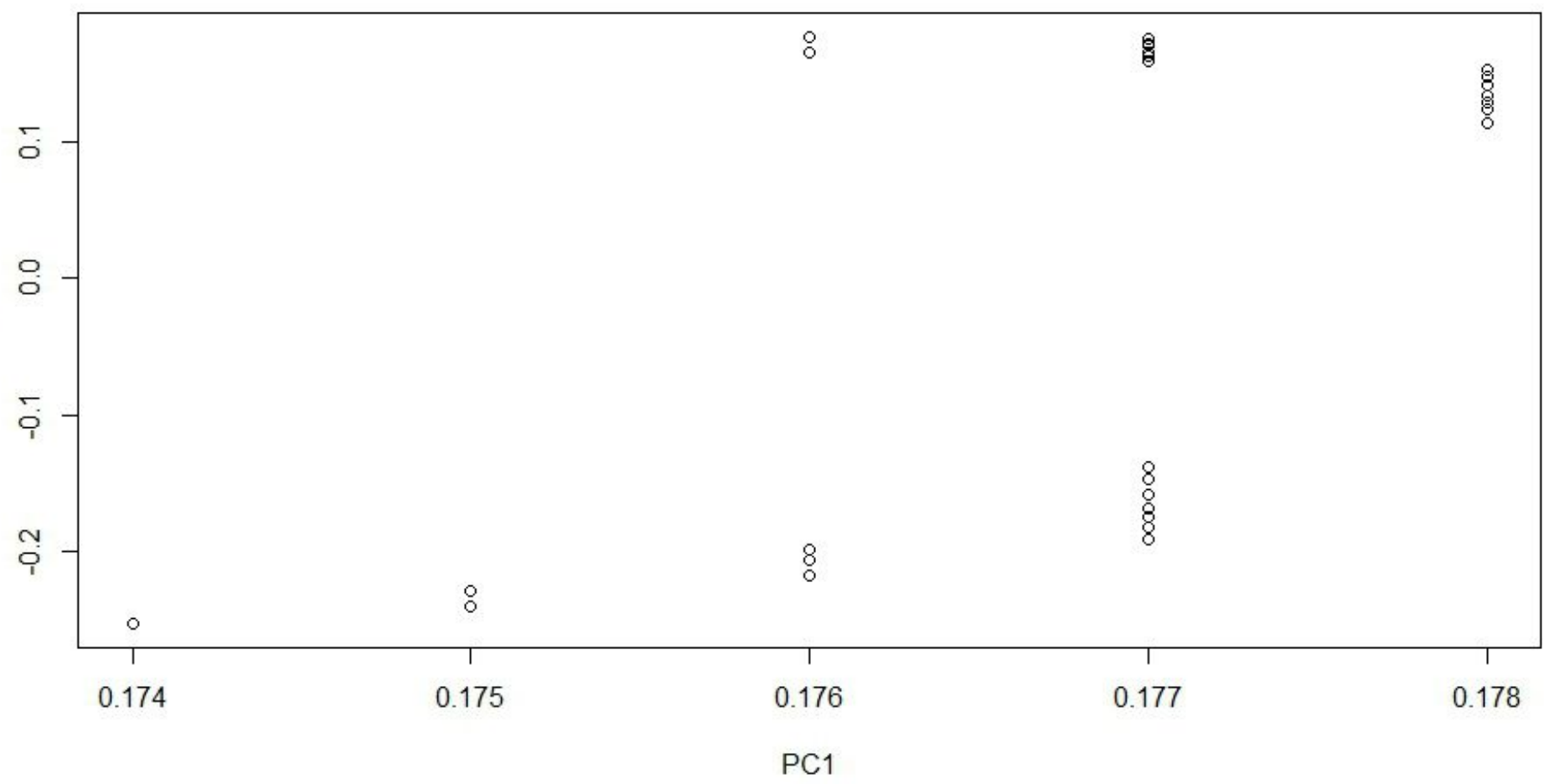

Figure 4

Components PCA Diagram of texture lables of peritumoral edema ADC map

rmodel

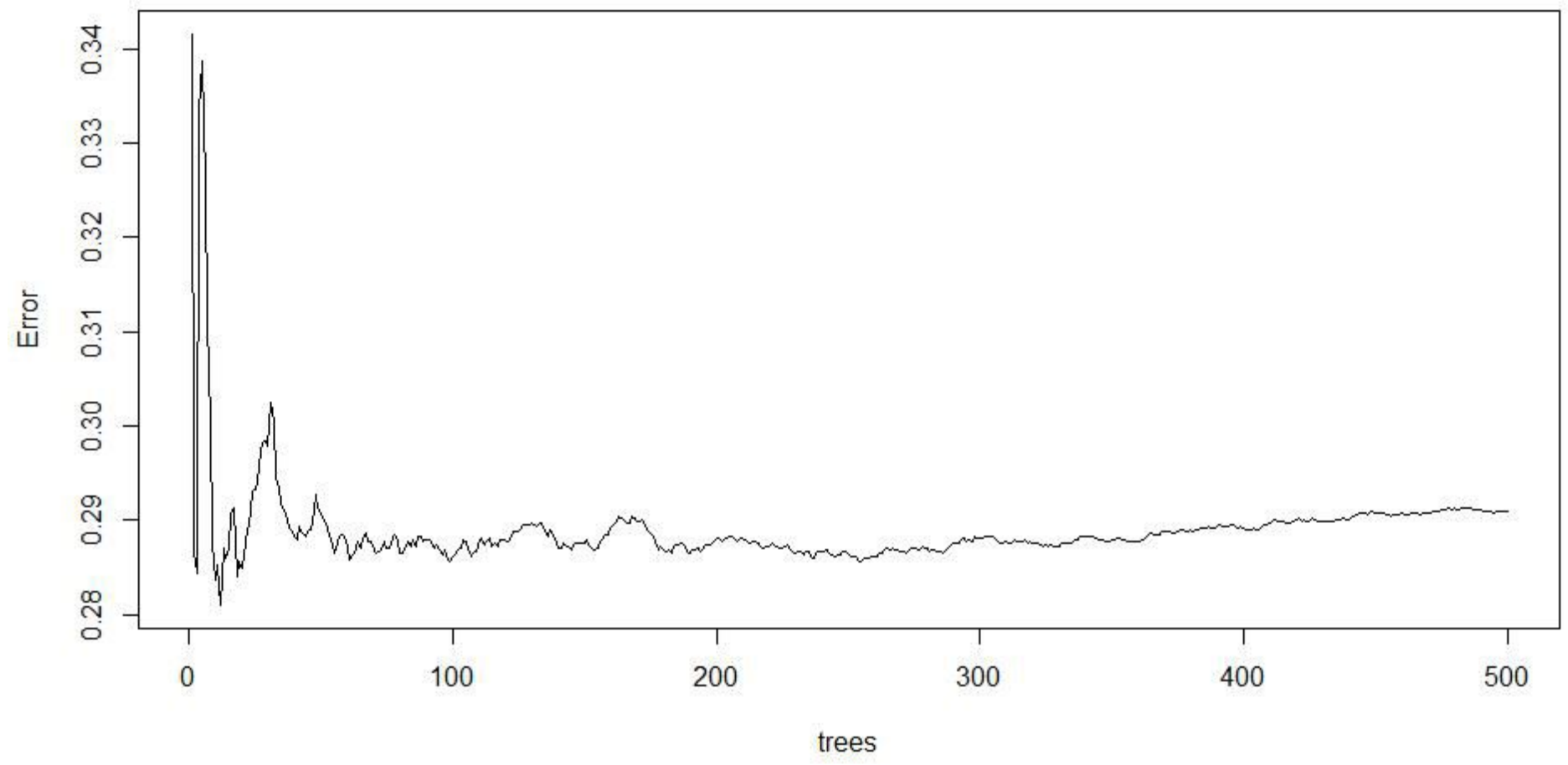

Figure 5 
Quantitative Error Graph for Selecting Random Trees of model (VASARI+TumorADC+preitumoral edemaADC)

\section{OOB ROC Curve Random Forest (VASARI+TumorADC+EdemaADC)}

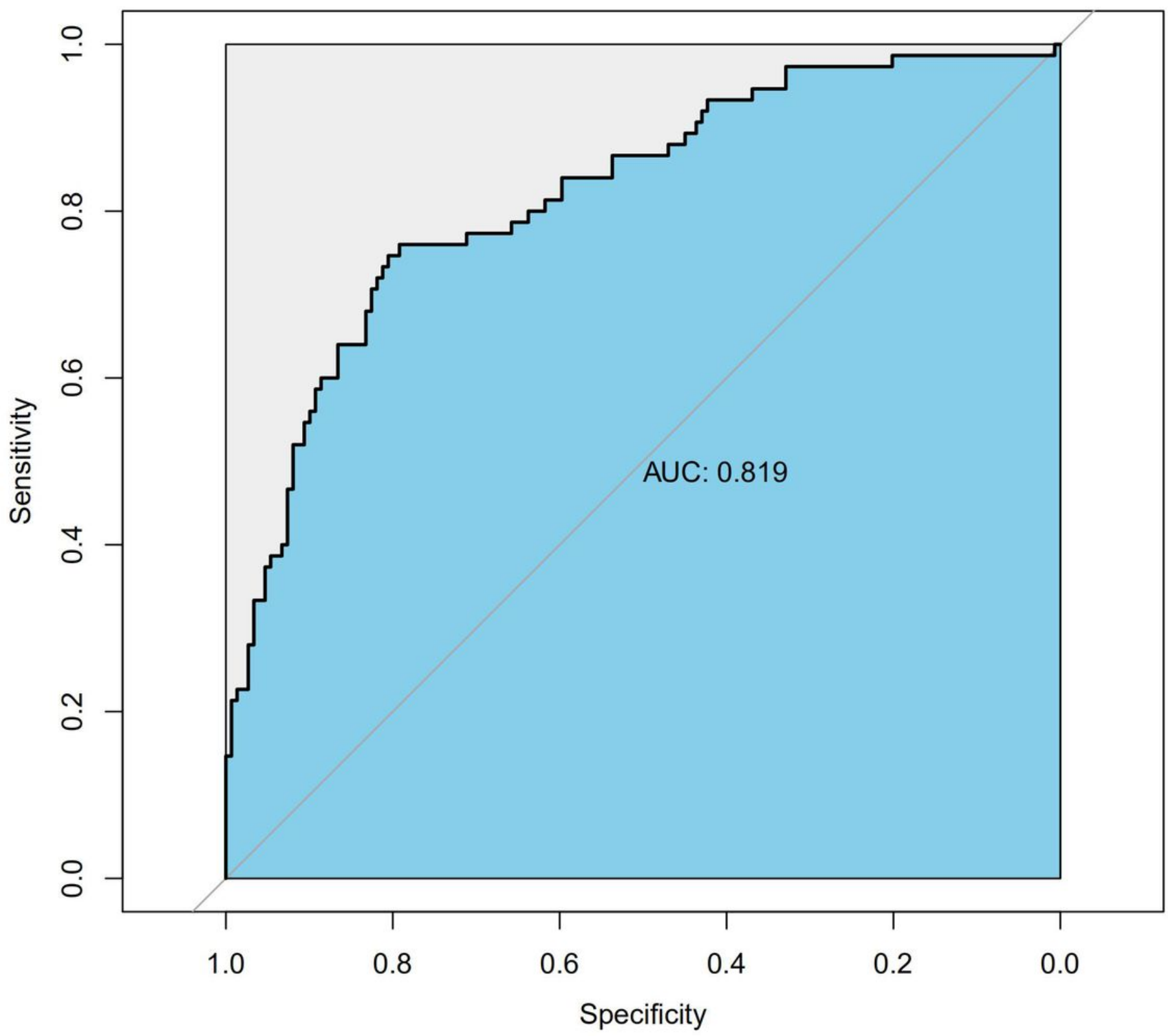

Figure 6

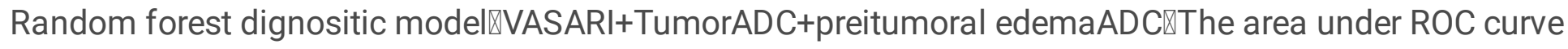
is $81.9 \%$. 


\section{Error Rates Random Forest individualize.csv}

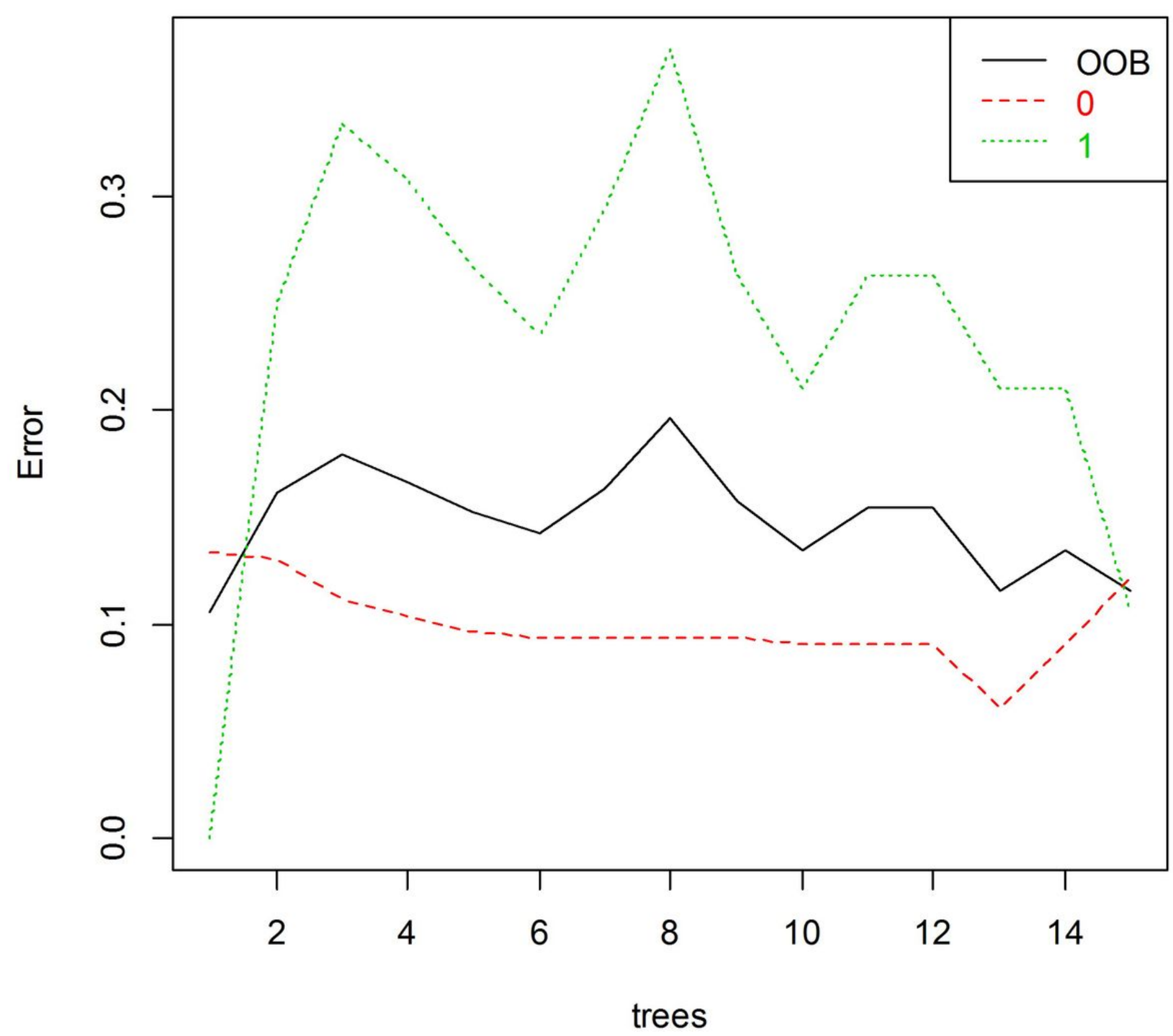

Figure 7

The error rate of model classification under different outcomes of stochastic forest model and the error rate of out-of-bag data classification of Stochastic Forest model. "0" represents the mutation of IDH1 gene and " 1 " represents the mutation of IDH1 gene. The error rate of this graph reflects the stability and scientificity of the final model (VASARI+TumorADC+preitumoral edemaADC). 